\title{
Performance of a Novel Energy-Efficient and Energy Awareness Scheme for Long-Lifetime Wireless Sensor Networks
}

\author{
Tan-Hsu Tan ${ }^{1}$, Neng-Chung Wang ${ }^{2}$, Yung-Fa Huang*, \\ Young-Long Chen ${ }^{3}$ and Yau-Der Wang ${ }^{1}$ \\ Department of Information and Communication Engineering, Chaoyang University of Technology, \\ 168, Jifeng E. Rd., Wufeng District, 413, Taichung, Taiwan \\ 'Department of Electrical Engineering, National Taipei University of Technology, \\ No. 1, ChungXiau E. Rd., Sec. 3, 106, Taipei, Taiwan \\ ${ }^{2}$ Department of Computer Science and Information Engineering, \\ National United University, 2, Lienda, Miaoli 36063, Taiwan \\ ${ }^{3}$ Department of Computer Science and Information Engineering, National Taichung University of \\ Science and Technology, 129, Shangmin Rd., Sec. 3, 404, Taichung, Taiwan
}

(Received July 2, 2014; accepted April 20, 2015)

Key words: wireless sensor networks, network lifetime, energy efficiency, cross layer, load balance

In wireless sensor networks (WSNs), multihop routing protocols can achieve energy efficiency for data aggregation. However, the network lifetime still suffers degradations owing to the unbalanced energy consumption for all nodes. Therefore, in this paper, with an energy-saving scheme, we propose an improved cross-layer algorithm for media access control (MAC) and routing protocols. The proposed cross-layer algorithm allows more nodes to share the load of the leader of data relay and balance the opportunity of data relay to all nodes, in which the proposed load-balancing scheme allocates the energy consumption load more uniformly among all nodes. Thus, the lifetime of the first node death (FND) can be clearly prolonged. An analysis is carried out to show the energy efficiency of the proposed cross-layer algorithm. Simulation results show that the proposed scheme can prolong the lifetime of WSNs to be double the lifetime of FND compared with that using the shortest hop routing tree (SHORT) scheme.

\section{Introduction}

The wireless sensor network (WSN) consists of a large amount of sensing nodes that carry out sensing, computing and communicating capabilities to perform data gathering for a target area. Owing to the small element of the sensing nodes, the computation and

"Corresponding author: e-mail: yfahuang@mail.cyut.edu.tw 
battery are limited to work for a long period.(1) Besides, the sensing area is always not easy to reach to charge the battery of the nodes. Then, the energy efficiency becomes one of the most important issues for WSNs. To achieve energy efficiency for data transmission, the multihop transmission is considered to be the main transmission scheme for WSNs. In the multihop scheme, most nodes need to sense and relay data to their neighbor nodes. The data fusion technique can integrate data from different sources and remove redundant data, thus decreasing the amount of transmission data to reduce energy consumption and lessen the transmission delay. ${ }^{(2,3)}$

Among the recent routing algorithms for WSNs, power-efficient gathering in sensor information systems (PEGASIS), power-efficient data gathering and aggregation protocol-power aware (PEDAPPA) and BINARY focus on the energy efficiency and prolonging the network lifetime. ${ }^{(4-6)}$ However, they suffer from severe transmission delay. Therefore, the shortest hop routing tree (SHORT) algorithm in ref. 7 was proposed for WSNs. The SHORT algorithms construct some synchronous communication pairs (CPs) with two nearest nodes and find the nearest nodes to relay the data. ${ }^{(7)}$ However, owing to the random deployments of nodes, some active nodes perform redundant data sensing and further increase delay. Thus, the media access control (MAC) layer protocol, namely, the turn-off redundant node (TORN) in ref. 8, allows redundant nodes to turn into a sleeping mode without degrading the network coverage and to improve the data transmission delay. ${ }^{(8)}$ Therefore, the combined schemes of SHORT and TORN (SHORTORN) can further compromise energy efficiency and transmission delay. However, owing to the increasing average distance between the nodes after the TORN, the first exhausting energy node suffers from lifetime shrinkage. ${ }^{(9)}$

Moreover, in SHORT, the leader node relaying data to a base station (BS) is selected by the node's remaining energy over the distance to a BS. The nearer node could be selected as the leader node, but oftentimes, its energy is still exhausted. Then, the lifetime of the first node death (FND) would be diminished. Therefore, to limit the number of times of being a leader node, a weight is proposed in the leader selection coefficient. Therefore, in this paper, we propose an energy-aware weight-based protocol (EAWP) to further prolong the network lifetime of the FND, to improve the performance of energy consumption, and to minimize the transmission delay of products.

The rest of this paper is organized as follows. In $\S 2$, we describe the transmission model in the WSNs. In $\S 3$, a proposed modified energy-aware MAC protocol is introduced to reduce the energy consumption of redundant nodes. Next, in $\S 4$, some improved cross-layer schemes are proposed to prolong the lifetime for SHORT schemes. In $\S 5$, a theoretical analysis is derived to verify the performance of the proposed crosslayer schemes. Some simulation results are obtained to show the improvement of the proposed schemes. Conclusions are finally drawn in $\S 7$.

\section{Transmission Models}

In this paper, the WSNs consist of a large number of identical sensing nodes, which are uniformly and randomly distributed in a sensing area. In the sensing area, a global positioning system (GPS) is assumed to obtain the position of the nodes for the BS. 
After the sensing nodes are deployed in the area, they are fixed and their battery cannot be charged or changed. Each node can adjust its transmission power to communicate with the other nodes.

Each active node sends a one $k$-bit packet to its neighbor node per round. The intermediate node receives data from other nodes and relays one packet obtained by data fusion to its neighbor node. In each round, any active node after sensing or gathering data always sends out a one $k$-bit packet via the CP. The data aggregation in the middle nodes receiving many packets generates a one $k$-bit packet, assuming perfect data fusion.

Owing to the limited energy in WSNs and the uncharged battery in the deployed nodes, efficiently utilizing the energy in the nodes is a very important issue for the WSNs. In the MAC layer, the periodical sleep is usually a scheme for saving energy. ${ }^{(10-12)}$ There are two activity periods: active mode and sleep mode. In the active mode, the sensors can communicate with the surrounding nodes. However, in the sleep mode, the sensor nodes stop communicating to save energy. Moreover, to further reduce energy consumption, the data fusion scheme is used to reduce the amount of redundant transmission data.

In the physical layer of the system model, the energy consumption for the amplifier circuit of transmitter and receiver is assumed to be $E_{\text {elec }}=50 \mathrm{~nJ} / \mathrm{bit}$. The transmission energy for the transmitter amplifier is assumed to be $\varepsilon_{\text {amp }}=100 \mathrm{pJ} / \mathrm{bit} / \mathrm{m}^{2}$. (2) Then, the energy requirement to transmit one packet of $k$ bits for a distance $\mathrm{d}$ can be expressed as

$$
E_{\mathrm{T}_{\mathrm{x}}}(k, d)=k E_{\text {elec }}+k d^{2} \varepsilon_{\mathrm{amp}},
$$

where $E_{\text {elec }}$ and $\varepsilon_{\text {amp }}$ are the energy consumptions of an electric circuit and a power amplifier of a transmitter, respectively. Moreover, the energy consumption to receive a one $k$-bit packet is expressed as

$$
E_{\mathrm{R}_{\mathrm{x}}}(k)=k E_{\text {elec }}
$$

\section{Modified Energy-Aware MAC}

The time division multiple access (TDMA) scheme is adopted in the MAC protocol. To achieve energy efficiency, the data is transmitted by multihop transmission, in which all farther nodes send their data via middle nodes toward the sink. Therefore, all the communication links between two nodes should use different time slots to avoid collision. Moreover, some synchronous CPs with two nearest nodes are constructed, and the time slots are firstly assigned for farther pairs. Then, the nearer CPs relay the data in the following time slot. Thus, the neighbor CPs do not use the same time slot.

Moreover, when there are several transmitters transmitting packets to the same node, the TDMA divides the time slots for the receiving node to avoid data collision. Owing to the limited energy of the nodes in WSNs and possible high density of node distribution, the sensing coverage of the nodes could overlap with each other. Thus, the sensing nodes redundant for sensing data can be turned into sleeping mode to save energy. ${ }^{(9,10)}$

In the TORN algorithm, all the nodes are initiated at the same time. The node's position can be detected by GPS and is broadcasted to the neighbor nodes. Then, all the 
nodes obtain the list of all their neighbor nodes sorted by distance from near to far. The list is called redundant neighbor list (RNL). ${ }^{(8)}$ After the sorting of the RNL, the nodes are determined to be in active or sleep mode by contention. When one node obtains its active mode, it broadcasts this information to turn off its neighbors in the sensing range (SR). The TORN algorithms can make the distribution of the nodes uniform and do not need to lower the density of the nodes. After executing TORN, the nodes will become active or sleep to effectively lessen the energy consumption.

Moreover, to efficiently perform data aggregation, three steps are adopted for efficient routing protocols:

(1) Only one leader node is elected to transmit the gathered data to the BS.

(2) The sensing data packets are transmitted to the leader node via the shortest route.

(3) The data relaying performs the most efficient data fusion technique to reduce data redundancy.

In transmission, some nodes could be repeatedly elected as the leader to consume the energy quickly. Thus, to balance the energy consumption, the leader should be evenly allocated to most of the nodes. ${ }^{(4,5)}$

In the SHORT algorithms, it is considered that the BS can manage the network topology, perform the data aggregation with a routing table, and schedule the time table.(7) To prolong the network lifetime, an energy coefficient for the leader selection, $\eta_{\mathrm{L}, \mathrm{S}}$, is defined. The BS selects the leader node among all the nodes on the basis of the maximal $\eta_{\mathrm{L}, \mathrm{S}}$. The energy coefficient is given by

$$
\eta_{\mathrm{L}, \mathrm{S}}=E_{\mathrm{r}} / d_{\mathrm{o}}^{2}
$$

where $E_{\mathrm{r}}$ and $d_{\mathrm{o}}$ are the remaining energy of the target node and the distance between the BS and the target node, respectively.

As the leader node is elected, the BS can manage the network topology and then inform the farther nodes to construct their CPs and schedule the time slots. With the assumption of the full management capability to calculate the routing path and time slot schedule in the BS, the amount of $\left\lfloor n / 2^{i}\right\rfloor$ CPs is the maximal number of pairs at the ith time slot for $n$ nodes of WSN. The least data delay will be $\left(\left[\log _{2} n\right\rceil+1\right)$ for a complete data transmission for $n$ nodes of WSN. Therefore, in each time slot of SHORT algorithms, the CPs select the best neighbor nodes to transmit data and obtain the best time delay and energy efficiency.

\section{Improved Cross-Layer Schemes}

At first, to investigate the energy efficiency of the SHORT algorithms, we perform 2100 computer simulations in WSNs. To realize the number of nodes as a leader $\left(N_{\mathrm{L}}\right)$ before the FND, we perform an experiment to find $N_{\mathrm{L}}$ and the average lifetime of FND, as shown in Table 1. From Table 1, it is clearly observed that the lifetime of FND is proportional to the $N_{\mathrm{L}}$. That is, the larger the $N_{\mathrm{L}}$, the longer the lifetime.

To further improve the load balancing of the leader for nodes in the SHORT algorithm, in the improved SHORT algorithm, we modify the energy coefficient for the leader selection, $\eta_{\mathrm{L}, \mathrm{IS}}$, by 
Table 1

Average lifetime for the number of nodes being a leader before FND.

\begin{tabular}{cccc}
\hline$N_{\mathrm{L}}$ & Average lifetime & $N_{\mathrm{L}}$ & Average lifetime \\
\hline 2 & 853 & 11 & 1608.9 \\
3 & 982.3 & 12 & 1681.4 \\
4 & 1066.1 & 13 & 1755.1 \\
5 & 1122.3 & 14 & 1802.4 \\
6 & 1204.3 & 15 & 1823.4 \\
7 & 1276.8 & 16 & 1896.7 \\
8 & 1386.3 & 17 & 1942.6 \\
9 & 1477.1 & 18 & 2029 \\
10 & 1540.4 & 19 & 2181.5 \\
& & 20 & 2221.5 \\
\hline
\end{tabular}

$$
\eta_{\mathrm{L}, \mathrm{IS}}=\left(C-N_{\mathrm{L}}\right) E_{\mathrm{r}} / d_{\mathrm{o}}^{2}
$$

where $E_{\mathrm{r}}$ and $d_{\mathrm{o}}$ are the same as in eq. (3) and $N_{\mathrm{L}}$ is the number of times a node has been a leader. The threshold of number of times of being a leader, $C$, in eq. (4) is used to balance the number of times of becoming a leader for nodes. Thus, when the $N_{\mathrm{L}}$ of a node increases, the factor $\left(C-N_{\mathrm{L}}\right)$ decreases the frequency of the node to be a leader. Moreover, when a node has become a leader $C$ times, the factor $\left(C-N_{\mathrm{L}}\right)$ will become zero to stop the node from being a leader. Moreover, if the factors $\left(C-N_{\mathrm{L}}\right)$ of all nodes become zero, the energy coefficient for the leader selection, $\eta_{\mathrm{L}}$, in eq. (3) will be used in the remaining lifetime for WSNs. The improved SHORT is called weight-based SHORT (WSHORT). Furthermore, to maintain the coverage and reduce the data delay, the TORN scheme is combined with the SHORT algorithms, and we call it the SHORTORN scheme.

In general, the nodes that have been a leader could be dead earlier than those that are never leaders owing to the high energy consumption. However, it is not always true. With one pattern of CPs in WSNs, in a CP, one node may transmit to the other node, which is not the nearest node for it. Therefore, when the number of patterns of CPs is not high, some nodes that never become a leader would be dead earlier than those that have been a leader owing to their long-distance CPs.

From eqs. (1) and (2), we know that the energy consumption in the nodes depends on not only the transmission distance but also the number of transmissions. Moreover, in a specific CP, all nodes would be transmitters or receivers. Then, the transmission distances of the nodes and the number of transmissions of nodes are determined. Thus, to balance the energy consumption of the nodes, the patterns of CPs should be diverse. Therefore, we further propose an energy-aware SHORT (ESHORT) scheme to prolong the lifetime of FND.

To balance the energy consumption of nodes in each round, we modified the construction procedure on CPs. In SHORT, the first nodes selected to construct the CP are the farthest nodes. The transmitters of the farthest nodes only need to transmit data 
once. Then, it would result in less energy consumption. Therefore, in the proposed weight-based SHORTORN (WSHORTORN), we further consider the remaining energy of the nodes. Then, the first node $\mathrm{nI}$ elected to construct the CP is obtained by

$$
I=\arg \max _{i} \frac{d_{i}}{e_{i}}
$$

where $d_{i}$ is the distance between the $i$ th node and the leader node, and $e_{i}$ is the remaining energy of the $i$ th node. Therefore, with the proposed weight-and-energy-aware scheme, the SHORT and TORN schemes can be combined into the energy-aware WSHORTORN (EWSHORTORN) scheme to improve the network lifetime.

\section{Approximated Analysis}

To verify the performance of the proposed SHORTORN cross-layer algorithms, we analyze approximately the energy consumption of SHORT, SHORTORN and WSHORTORN algorithms. In $\S 4$, we know that the SHORT scheme exerts three data transmission models for the nodes. The first type of node is $u_{\mathrm{T}}$, which only transmits data to its neighbors, but does not receive any data. The second type of node is $u_{\mathrm{TR}}$, which not only transmits data to its neighbors, but also receives data. The third type of node is the leader, $u_{\mathrm{L}}$, which receives the relaying data from neighbors and transmits them to the BS.

We analyze the energy consumption of the three types of node. In the network, there are about $[N / 2\rceil$ nodes. Thus, the node $u_{\mathrm{T}}$ consumes energy as

$$
\bar{E}_{\mathrm{T}}=\left\lceil\frac{N}{2}\right\rceil\left(k E_{\text {elec }}+k \varepsilon_{\mathrm{amp}} \bar{d}_{\mathrm{utu}}^{2}\right)
$$

where $\bar{d}_{\text {utu }}$ is the average distance between the nodes and the nearest-neighbor nodes. The number of the node $u_{\mathrm{TR}}$ can be obtained by $N-\lceil N / 2\rceil-1$. Then, the total energy consumption of the node $u_{\mathrm{TR}}$ is calculated as

$$
\bar{E}_{\mathrm{TR}}=\left(N-\left\lceil\frac{N}{2}\right\rceil-1\right)\left(k E_{\text {elec }}+k \varepsilon_{\text {amp }} \bar{d}_{\text {utu }}^{2}\right)+(N-1) \cdot k E_{\text {elec }} .
$$

Moreover, the energy consumption of only one leader can be obtained as

$$
\bar{E}_{\mathrm{L}}=k E_{\mathrm{elec}}+k \varepsilon_{\mathrm{amp}} \bar{d}_{\mathrm{LtB}}^{2},
$$

where $\bar{d}_{\mathrm{LtB}}$ is the distance between the leader and the BS. Then, the total energy consumption of $N$ nodes for SHORT algorithms in WSNs can be obtained by summing the energy consumption of these three types of node: $u_{\mathrm{T}}, u_{\mathrm{TR}}$, and $u_{\mathrm{T}}$ as

$$
\bar{E}_{\mathrm{S}}=(N-1)\left(k E_{\text {elec }}+k \varepsilon_{\text {amp }} \bar{d}_{\mathrm{utu}}^{2}\right)+N \cdot k E_{\text {elec }}+k \varepsilon_{\mathrm{amp}} \bar{d}_{\mathrm{LtB}}^{2} \text {. }
$$


To find the distance statistics among the nodes, we assume that the nodes are uniformly distributed in WSNs, as shown in Fig. 1. If there are total $N$ nodes, the average area covered by one node is $L^{2} / N$, which can be assumed to be a square by $(L / \sqrt{N}) \times(L / \sqrt{N})$, as shown in Fig. 1 .

With the assumption of the data transmission being performed only for nearest neighbors, node 1 only transmits data to node 2 or node 3 in Fig. 1 . Thus, we can find the expectation of the $x$-axis and $y$-axis of node 1 as $E\left[x_{1}\right]=E\left[y_{1}\right]=L /(2 \sqrt{N})$. Moreover, the variances for $x_{1}$ and $y_{1}$ can be obtained as $E\left[x_{1}^{2}\right]=E\left[y_{1}^{2}\right]=L^{2} /(3 N)$. Similarly, the mean value and variance for the axis of node 3 can be found using $E\left[x_{3}\right]=3 L /(2 \sqrt{N})$, $E\left[y_{3}\right]=L /(2 \sqrt{N}), E\left[x_{3}^{2}\right]=7 L^{2} /(3 N)$ and $E\left[y_{3}^{2}\right]=L^{2} /(3 N)$. Then, the average distance between the neighbor nodes is obtained as

$$
\bar{d}_{\mathrm{utu}}^{2}=E\left[\left(x_{1}-x_{3}\right)\left(y_{1}-y_{3}\right)\right]=\frac{4 L^{2}}{3 N} .
$$

To find the distance between the leader node $\left(x_{\mathrm{L}}, y_{\mathrm{L}}\right)$ and the BS $\left(x_{\mathrm{BS}}, y_{\mathrm{BS}}\right)$, owing to the leader selection coefficient $E_{\mathrm{r}} / d_{\mathrm{LtB}}^{2}$, we consider that the leader nodes are distributed in the front 1/5 area, as shown in Fig. 2. Then, the mean position of the leader nodes can be obtained using $E\left[x_{\mathrm{L}}\right]=L / 2$ and $E\left[y_{\mathrm{L}}\right]=9 L / 10$. The variance of the position of leader nodes is obtained using $E\left[x_{\mathrm{L}}^{2}\right]=L^{2} / 3$ and $E\left[y_{\mathrm{L}}^{2}\right]=61 L^{2} / 75$. Therefore, the variance of the average distance from the leader to the BS can be obtained as

$$
\begin{aligned}
\bar{d}_{\mathrm{LtB}}^{2} & =E\left[\left(x_{\mathrm{BS}}-x_{\mathrm{L}}\right)^{2}+\left(y_{\mathrm{BS}}-y_{\mathrm{L}}\right)^{2}\right] \\
& =\left(x_{\mathrm{BS}}-\frac{\sqrt{3}}{3} L\right)^{2}+\left(y_{\mathrm{BS}}-\frac{\sqrt{183}}{15} L\right)^{2}+L\left[\left(\frac{2 \sqrt{3}}{3}-1\right) x_{\mathrm{BS}}+\left(\frac{2 \sqrt{183}}{15}-\frac{9}{5}\right) y_{\mathrm{BS}}\right] .
\end{aligned}
$$
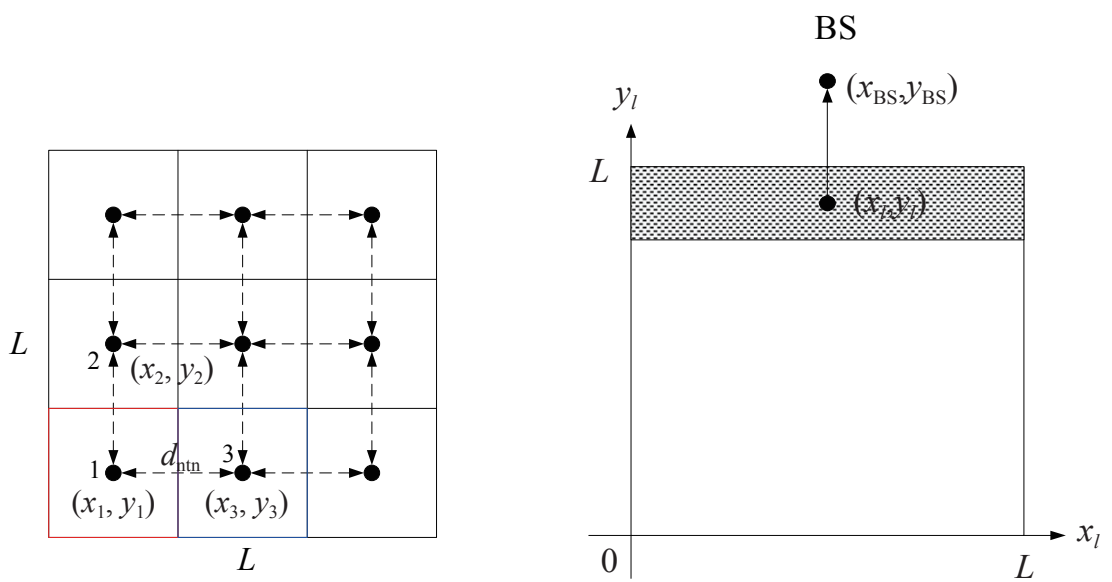

Fig. 1 (left). Uniformly distributed nodes in WSNs.

Fig. 2 (right). Uniformly distributed area of the leader nodes for SHORT and SHORTORN in WSNs. 
Next, in the SHORTORN routing algorithm, part of the redundant nodes enter the sleep mode and consume no energy. Assume that there are $N_{\mathrm{s}}$ redundant nodes in total $N$ nodes of WSNs. Then, the total energy consumption of SHORTORN can be obtained as

$$
\bar{E}_{\mathrm{ST}}=\left(N-N_{\mathrm{s}}-1\right)\left(k E_{\text {elec }}+k \varepsilon_{\mathrm{amp}} \bar{d}_{\mathrm{utu}}^{2}\right)+\left(N-N_{\mathrm{s}}\right) \cdot k E_{\text {elec }}+k \varepsilon_{\mathrm{amp}} \bar{d}_{\mathrm{LtB}}^{2}
$$

Moreover, the total energy consumption of the proposed WSHORTORN algorithm can be expressed as

$$
\bar{E}_{\mathrm{ST}}=\left(N-N_{\mathrm{s}}-1\right)\left(k E_{\text {elec }}+k \varepsilon_{\mathrm{amp}} \bar{d}_{\mathrm{utu}}^{2}\right)+\left(N-N_{\mathrm{s}}\right) \cdot k E_{\text {elec }}+k \varepsilon_{\mathrm{amp}} \bar{d}_{\mathrm{L}^{\prime} \mathrm{tB}}^{2},
$$

where $\bar{d}_{\mathrm{L}^{\prime} \mathrm{B}}$ is the average distance from the leader nodes to the BS for WSHORTORN. Thus, the difference between SHORTORN and WSHORTORN is the leader node selection coefficient. In WSHORTORN, we let most nodes obtain the probability to be a leader. Therefore, the coverage area of the leader nodes for WSHORTORN can be uniformly expanded to an entire area in WSNs, as shown in Fig. 3. Thus, the axis statistics for the leader nodes $\left(x_{\mathrm{L}^{\prime}}, y_{\mathrm{L}^{\prime}}\right)$ can be obtained using $E\left[x_{\mathrm{L}^{\prime}}\right]=L / 2$ and $E\left[y_{\mathrm{L}^{\prime}}\right]=L / 2$. Similarly, the variance of the position of leader nodes is obtained using $E\left[x_{L^{2}}^{2}\right]=L^{2} / 3$ and $E\left[y_{\mathrm{L}^{\prime}}^{2}\right]=L^{2} / 3$. Consequently, in the WSHORTORN, the variance of the average distance from the leaders to the BS is obtained as

$$
\begin{aligned}
\bar{d}_{\mathrm{L}^{\prime} \mathrm{B}}^{2} & =E\left[\left(x_{\mathrm{BS}}-x_{\mathrm{L}^{\prime}}\right)^{2}+\left(y_{\mathrm{BS}}-y_{\mathrm{L}^{\prime}}\right)^{2}\right] \\
& =\left(x_{\mathrm{BS}}-\frac{\sqrt{3}}{3} L\right)^{2}+\left(y_{\mathrm{BS}}-\frac{\sqrt{3}}{3} L\right)^{2}+L\left(\frac{2 \sqrt{3}}{3}-1\right)\left(x_{\mathrm{BS}}+y_{\mathrm{BS}}\right) .
\end{aligned}
$$

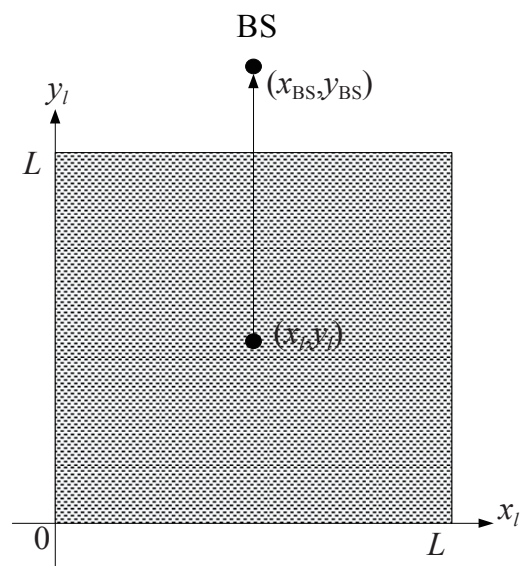

Fig. 3. Distribution area of leader nodes in WSHORTORN routing algorithms. 


\section{Simulation Results}

To verify the performance of the WSN lifetime prolonging scheme, computer simulation by Matlab programming is performed for SHORT, TORN and SHORTORN schemes. In our simulation, the sensor nodes are uniformly distributed on a square area located at $(0,0)-(100,100)$ with $100 \times 100 \mathrm{~m}^{2}$. The BS is deployed at $(50,150)$. In all TORN schemes, the sensing range (SR) is set to $10 \mathrm{~m}$ to perform sufficiently high coverage for WSN. In all the nodes, the initial energy is all one joule. The perfect power control scheme to perform no error pack transmission is assumed for all the nodes. The length of one packet is 2000 bits, and the energy consumptions of transmission and reception are modeled using eqs. (1) and (2), respectively. The nodes are deployed randomly 30 times to determine the sufficient statistics of performance in WSNs. As for the proposed SHORTORN schemes, the redundant nodes remain in the sleep mode until the active nodes are dead.

In WSHORTORN, the weight is set by $C=100$. To compare the energy efficiency, the energy consumption for each round is defined as the total energy consumption divided by the lifetime of FND. The number of nodes is from 50 to 300 . Then, the numerical results for SHORT calculated using eq. (9) are compared with the simulation results shown in Fig. 4. From Fig. 4, it is observed that our analysis and simulation results are in agreement. Moreover, from eq. (9), the energy consumption of SHORT is approximately proportional to the number of nodes N, as shown in Fig. 4.

In the comparison for SHORTORN, from Fig. 5, it is observed that the analytical results seem to be more optimistic than the simulation results owing to the distribution area of the leader nodes, especially for a higher density of nodes. Besides, when the number of nodes increases, the number of sleep nodes, $N_{\mathrm{s}}$, also increases. Therefore, from the analysis using eq. (12) and the comparison of Figs. 4 and 5, the energy consumption of SHORTORN is considerably less than that of SHORT, especially for a
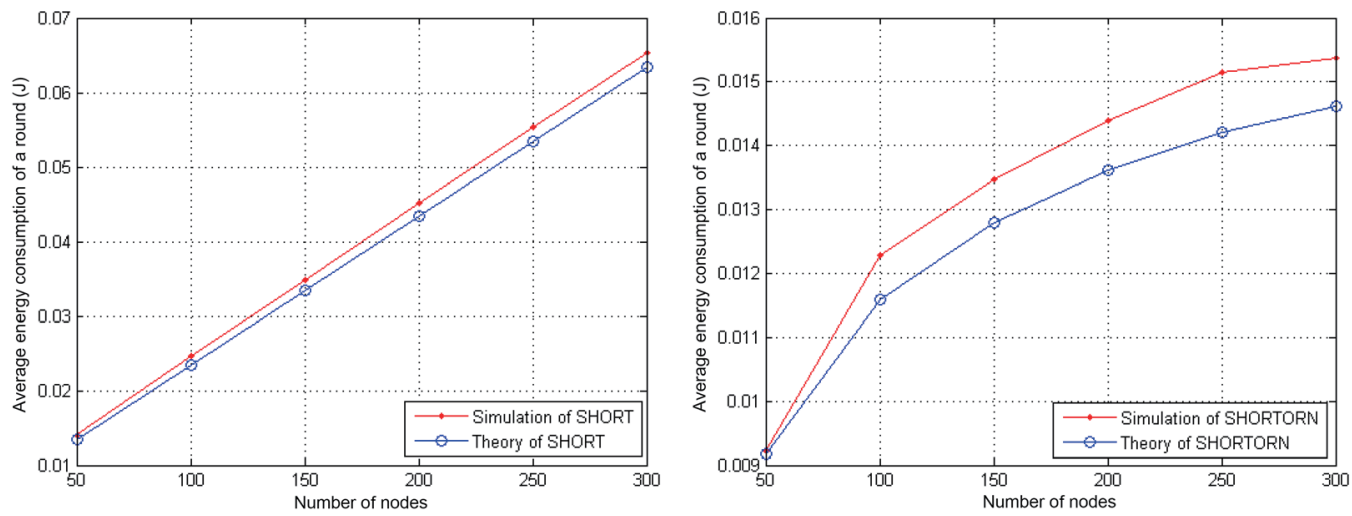

Fig. 4 (left). (Color online) Average energy consumption per round for SHORT.

Fig. 5 (right). (Color online) Average energy consumption per round for SHORTORN. 
large number of nodes. Moreover, with eq. (13), the comparison with WSHORTORN shown in Fig. 6 reveals that the analysis is more pessimistic than the simulation results since the leaders are assumed to be distributed in the entire area. In the comparisons of SHORTORN and WSHORTORN in Figs. 5 and 6, the energy consumption per round of WSHORTORN is slightly higher than that of SHORTORN. That is because in WSHORTORN, the energy consumption should be balanced with the constraint on the selection of leader nodes, and it results in more energy consumption of the leader nodes.

To further verify the performance improvement on prolonging the WSN lifetime, we simulate 50 sensor nodes uniformly distributed on a square area located at $(0,0)-(100$, 100 ) with $100 \times 100 \mathrm{~m}^{2}$. The improvement of different weight $C$ values for SHORTORN schemes is shown in Fig. 7. From Fig. 7, it is observed that the proposed SHORTORN can prolong the lifetime of 40,50 , and $100 \%$ of ND. However, since fewer nodes are active in SHORTORN, the mean distance of the CPs is longer than that of SHORT. Then, in SHORTORN, the lifetime of FND is shorter than that in SHORT.

After the energy coefficient for the leader selection, $\eta_{\mathrm{L}}$, is modified using eq. (4), the lifetime of the FND of the WSHORTORN is improved to outperform those of the SHORT and SHORTORN schemes, as shown in Table 2. From Table 2, it is observed that with $C=100$, the lifetime of FND is the best, but with $C=1000$, the lifetimes of 30 and $50 \%$ of ND are the best. Therefore, from the results of SHORTORN shown in Fig. 7 , nodes could be selected to be a leader node too many times until their energies are exhausted. Then, the lifetime of the FND would be diminished. Therefore, to balance the energy consumption of nodes, we limit the number of times of being a leader for the node; the WSHORTORNs can considerably prolong the lifetime of the FND in comparison with SHORT.

Moreover, in this paper, EAWP schemes are used to further prolong the network lifetime of the FND. We compare the network lifetime performance of proposed SHORTORNs over the SHORT scheme for $C=100$, as shown in Fig. 8. From the
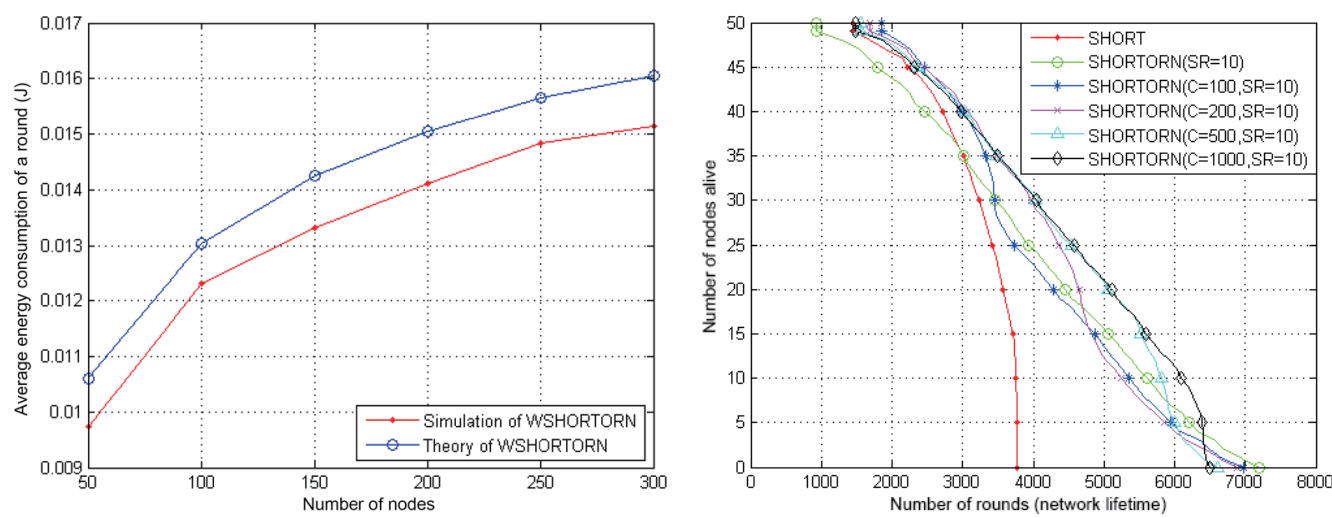

Fig. 6 (left). (Color online) Average energy consumption per round for WSHORTORN. Fig. 7 (right). (Color online) Lifetime performances for the SHORT and SHORTORN schemes. 
Table 2

Lifetime comparisons with different $\mathrm{C}$ values for WSHORTORN schemes.

\begin{tabular}{lrrrr}
\hline & \multicolumn{4}{c}{ Nodes } \\
\cline { 2 - 5 } Protocol & \multicolumn{1}{c}{$\%$} & $30 \%$ & $50 \%$ & $100 \%$ \\
\hline SHORT & 1447 & 3010 & 3416 & 3766 \\
SHORTORN & 933 & 3018 & 3941 & 7206 \\
WSHORTORN $(C=100)$ & 1857 & 3323 & 3726 & 6970 \\
WSHORTORN $(C=200)$ & 1689 & 3474 & 4365 & 6877 \\
WSHORTORN $(C=500)$ & 1561 & 3465 & 4547 & 6601 \\
WSHORTORN $(C=1000)$ & 1482 & 3486 & 4575 & 6502 \\
\hline
\end{tabular}

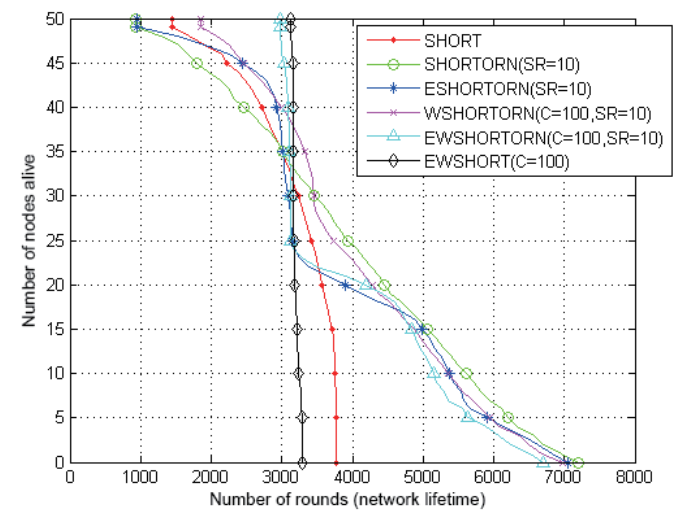

Fig. 8. (Color online) Lifetime performances for the SHORTORN and proposed EWSHORTORN schemes with weight $C=100$.

improvement in the performance of the proposed EWSHORTORN in Fig. 8, it is observed that the proposed EWSHORTORN can largely prolong the lifetime of $2 \%$ of ND. Moreover, the EWSHORT obtains the longest lifetime of FND among the schemes with total energy balancing and without the TORN scheme.

\section{Conclusions}

In this study, to improve the balance energy consumptions for nodes, we select a maximal number of times of being a leader to improve the SHORTORN crosslayer protocol. An approximated analysis is developed for the energy efficiency for SHORT schemes in WSNs. Numerical and simulation results show that the proposed WSHORTORN not only prolongs the network lifetime but also improves the energy efficiency. Furthermore, to balance the energy consumption of the nodes, we diverge the patterns of CPs with the remaining energy in the nodes, and then propose EWSHORTORN schemes to prolong the lifetime of FND. Simulation results show that the proposed EWSHORTORN largely prolongs the network lifetime of FND. 


\section{Acknowledgements}

This work was supported in part by the Ministry of Science and Technology (MOST), TAIWAN, under Grant Nos. 103-2221-E-324-018 and 103-2632-E-324-001-MY3.

\section{References}

1 D. Culler, D. Estrin and M. Srivastava: IEEE Comp. 37 (2004) 41.

2 K. Sohrabi, J. Gao, V. Ailawadhi and G. J. Pottie: IEEE Pers. Commun. 7 (2000) 16.

3 S. Lindsey, C. Raghavendra and K. M. Sivalingam: IEEE Trans. Parallel Distrib. Syst. 13 (2002) 924.

4 J. Culpepper, L. Dung and M. Moh: ACM SIGMOBILE Mobile Comp. Commun. Rev. 8 (2004) 61.

5 W. B. Heinzelman, A. P. Chandrakasan and H. Balakrishnan: IEEE Trans. Wireless Commun. $1(2002) 660$.

6 S. M. Jung, Y. J. Han and T. M. Chung: Proc. IEEE 9th Int. Conf. Adv. Commun. Technol. (2007) pp. 260-265.

7 Y. Yang, H. H. Wu and H. H. Chen: Proc. IEEE ICC (2006) pp. 3450-3454.

8 B. H. Liu, N. Bulusu, H. Pham and S. Jha: Proc. IEEE Globecom (2004) pp. 33-38.

9 W. Heinzelman, A. Chandrakasan and H. Balakrishnan: Proc. IEEE 33rd Annu. Hawaii Int. Conf. Sys. Sci. (2000) pp. 1-10.

10 C. Ma, M. Ma and Y. Yang: Proc. IEEE ICC (2004) pp. 3652-3656.

11 Y. Li, W. Ye and J. Heidemann: Proc. IEEE WCNC (2005) pp. 676-682.

12 D. Estrin, J. Heidemann and W. Ye: Proc. IEEE INFOCOM (2002) pp. 1567-1576. 